\title{
Quality of Service Provisioning in LTE-EPON Integrated Networks
}

\author{
Carlos A. Astudillo, Nelson L. S. da Fonseca (Advisor), \\ Juliana Freitag Borin (Co-advisor) \\ ${ }^{1}$ Institute of Computing - State University of Campinas (UNICAMP) \\ Avenida Albert Einstein, 1251 - 13.083-852 - Campinas - SP - Brazil \\ castudillo@lrc.ic.unicamp.br, \{nfonseca, juliana\}@ic.unicamp.br
}

\begin{abstract}
Passive optical networking (PON) has been recognized as a solution for backhauling next generation cellular networks. In this new scenario, QoS provisioning is an important challenge. This article proposes a Quality of Service $(Q o S)$ provisioning framework for LTE with EPON-based backhauling, which improves performance by taking advantage of information available from the backhaul as well as from the radio interface. It also introduces a QoS-aware LTE scheduler that dynamically adjusts users' priorities to increase the overall throughput. Experimental results show how the proposals can improve resource utilization and quality of service provisioning.
\end{abstract}

\section{Introduction}

In the past few years, users' demand of bandwidth and the need to meet strict Quality of Service (QoS) requirements in cellular networks have increased significantly. This scenario is driven by [Cisco 2014]: (i) the number of mobile users and mobile devices, such as tablets, laptops and smartphones, which have exponentially grown in the past years and such trend will continue for next years; $(i i)$ the amount of video traffic, which has reached $70 \%$ of the mobile Internet traffic; and (iii) the changes mobile Internet have introduced in the habits of users who are accessing the Internet seamlessly to complete multiple daily activities.

This situation has led Mobile Network Operators (MNOs) to implement the Long Term Evolution (LTE) technologies and to significantly increase the number of base stations. Since LTE base stations, evolved NodeBs (eNBs) in the LTE jargon, can offer peak downlink rates of $100 \mathrm{Mb} / \mathrm{s}$ in LTE and $1 \mathrm{~Gb} / \mathrm{s}$ in LTE-Advanced as well as half of these values in the uplink direction, they will inject a large amount of traffic into the Mobile Backhaul (MBH) network. Although the cost of the future MBH will be high, it can be reduced by using already deployed Fiber to Anywhere (FTTx) systems based on Passive Optical Networks (PONs), in which Ethernet PON (EPON) is the dominant technology in the market. Motivated by this, an attractive solution is to use EPONs as backhaul for the LTE technology.

In this new scenario, an LTE base station is a subscriber of the PON, leading to a new network architecture, called LTE-EPON, in which the Optical Network Unit (ONU) and the eNB can be integrated in a single device, called ONU-eNB. Since the ONU-eNB competes for bandwidth with other ONUs, the bandwidth given to the ONU-eNB can be lower than that needed to support the mobile users in the LTE network. Given this 
limitation in the available backhaul capacity, the support of QoS requirements of mobile users can be jeopardized.

This article presents the work in the dissertation [Astudillo 2015], which addresses the problem of QoS provisioning in LTE-EPON integrated networks and introduces the following original contributions:

- A novel QoS management framework for LTE/EPON networks. This framework is the first to describe how the ONU-eNB devices can improve performance by taking advantage of information sharing between EPON and LTE technologies.

- A new class of LTE schedulers, named backhaul-aware schedulers, that allocates radio resources considering information not only from the radio interface but also from the backhaul. The first backhaul-aware scheduler ever proposed was introduced in the dissertation.

- An uplink packet scheduler for QoS provisioning in LTE networks. The original contribution of this scheduler is the dynamic prioritization of users to increase the overall throughput of the system.

These contributions advance the state of the art in the design of integrated fiberwireless (FIWI) access networks, which is expected to be the predominant technology for next generation Internet access networks. This technology takes advantage of both the mobility and ubiquity of the wireless systems and the high capacity of the optical networks. The relevance of the original contributions in this paper is, therefore, to contribute to the evolution of Internet access technology which is so fundamental to our daily life. Currently, the bandwidth bottleneck of the Internet is located at the access network, which prevents Internet users to have greater access to high demanding applications and services based on video. We postulate that the impact of these contributions is to support the increasing demand for mobile Internet access, by meeting QoS requirements of the different users' applications. If the MNOs do not cope with the increase of number of users (as well as their demand), users" experience in the "connected world" will be quite compromised. The solutions for these problems proposed in [Astudillo 2015] provide less resource utilization at reduced costs since they use under-provisioned backhaul links and radio resources efficiently.

\section{Related Work}

This section briefly reviews the literature related to QoS provisioning in LTE networks and LTE-PON integrated networks, including the use of backhaul information in LTE networks. Existing QoS-aware schedulers for LTE networks [Anas et al. 2008, Delgado and Jaumard 2010, Marwat et al. 2014] prioritize Guaranteed Bit Rate (GBR) over non-GBR (nGBR) bearers by adopting different priority queues for each type of bearer. This can yield unnecessary loss of nGBR requests, decreasing the total throughput of the system. Moreover, all of these schedulers assume that the GBR parameter should be guaranteed to all traffic flows, which violates the LTE specification since non-GBR bearers have no rate guarantees.

On the other hand, few QoS provisioning schemes for cellular networks using PON-based backhaul can be found in the literature. Chung et al. [Chung et al. 2008] proposed a scheme for the two-upstream-wavelength PON (2W-PON) architecture in which real-time and control packets are prioritized by transmitting them on one wavelength 
while the low priority traffic is sent on another wavelength. However, 2W-PON architecture is not common in current deployed networks. Some QoS mapping schemes between LTE and PON have been proposed [Lim et al. 2012], [Chung et al. 2008], but with these approaches, the traffic in the EPON network can impact on the QoS provisioning of mobile users. The impact of available backhaul link capacity on performance of LTE networks has received little attention in the literature. In [Ghimire and Rosenberg 2014], the effect of limited backhaul capacity on the scheduling performance is studied. However, scheduling mechanisms that can deal with under-provisioned backhaul links are not reported in the literature.

In summary, there is no other work in the literature that proposes the use of backhaul information in LTE schedulers. Moreover, none of the above mentioned papers addresses the issue of coping with receiving less than the requested bandwidth. In addition, the dynamic prioritization of bearers to improve throughput was not explored before.

\section{Proposed Scheduler for QoS Provisiong in LTE Networks}

Although the focus of the present work is the QoS provisioning problem in LTE-EPON integrated networks, the LTE packet scheduler is essential for guaranteeing QoS requirements to mobile users and for optimally using scarce radio resources. The dissertation [Astudillo 2015] proposed the Z-Based QoS Scheduler (ZBQoS), an standardcompliant LTE uplink scheduler that provides QoS guarantees and improves overall network throughput. The ZBQoS scheduler dynamically modifies the priority of mobile users to favor those with strict QoS requirements over other users, when there are requests with delays close to the deadlines. However, when real-time traffic can be delayed (i.e., a request can wait a while to be served, but still respecting their QoS requirements), nonreal-time traffic can be served in order to increase the network throughput. Section 3.2 in [Astudillo 2015] gives a detailed description of the ZBQoS algorithm and its scheduling metrics which allow the dynamic prioritization of bearers.

We used the LTE-Sim simulator version 4.0 to evaluate the performance of the ZBQoS scheduler. The performance of the proposed LTE uplink scheduler was compared to that of a recently proposed scheduler called Bandwidth and QoS Aware (BQA) [Marwat et al. 2014], which is not standard compliant. The simulation scenarios are composed of a single cell, one eNB and several User Equipment (UE) devices (varying from 5 to 150 , with increments of 5). Users are uniformly distributed, transmitting Voice-overIP (VoIP), video or Constant Bit Rate (CBR) traffic. VoIP and video traffic are transmitted using GBR bearers and CBR (best effort) traffic uses nGBR bearers. Table 3.1 and 3.2 in [Astudillo 2015] give a complete description of simulation parameters, traffic models, QoS requirements and assumptions employed in the simulations.

The ZBQoS scheduler produces lower Packet Loss Ratio (PLR) values for CBR traffic when the network is overloaded but slightly higher PLR values when the network is heavily loaded (Fig. 1a). However, the lower PLR given by the BQA scheduler in heavyload regime is achieved at the expense of the QoS provisioning for video traffic (Fig. 1b). Although the maximum acceptable PLR value for video traffic is $1 \%$, the values produced by BQA scheduler increase with the traffic load, reaching $15 \%$ under heavy load. Thus, only the proposed scheduler can provide QoS guarantees to real-time traffic under all traffic conditions. Subsection 3.4.2 in [Astudillo 2015] presents additional results. 


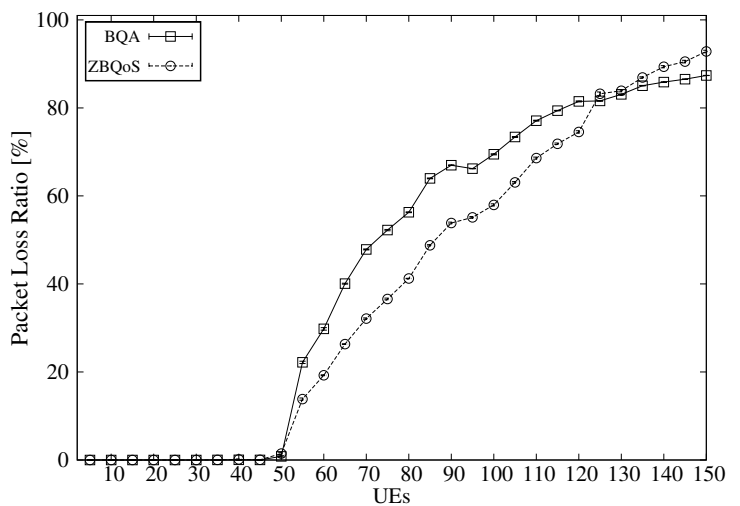

(a) CBR traffic

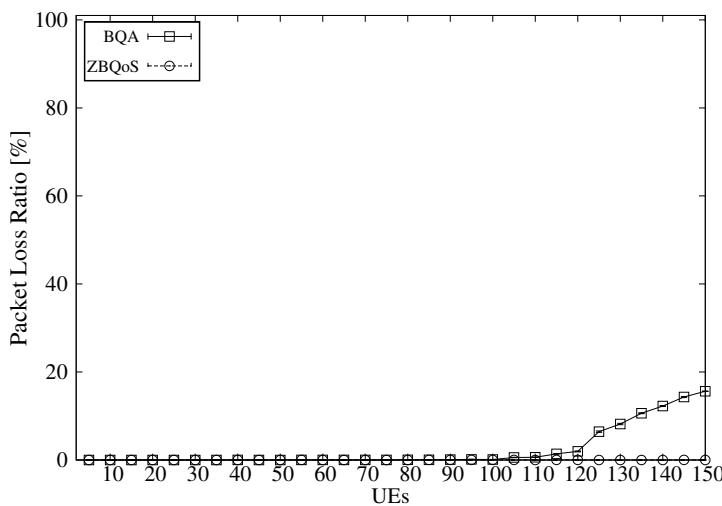

(b) Video traffic

Figure 1. Performance of the ZBQoS scheduler

\section{Proposed Framework for QoS Provisioning in LTE-EPON Integrated Networks}

The previous section proposed a novel LTE uplink scheduler for QoS provisioning, which performs dynamic prioritization of mobile users to improve performance. However, the proposed scheduler and those existing in the literature do not take into account the state of the backhaul link to allocate radio resources, which can potentially degrade QoS provisioning for mobile users in overload conditions as well as waste both radio and backhaul resources. To cope with the variability of the backhaul link capacity and provide QoS requirements to mobile users in an integrated LTE-EPON network, this section introduces a framework that improves QoS provisioning for mobile users of LTE networks by taking advantage of the capacity variability of EPON-based backhaul links.

The framework provides a method for modifying existing schedulers so that the backhaul load can be considered to allocate radio resources. It also includes the functional architecture of the ONU-eNB integrated device and a QoS mapping between LTE and EPON networks. This framework can be rapidly adopted in current and future deployments, capitalizing in the already deployed PON systems.

The proposed method is based on detecting when the ONU-eNB receives less bandwidth than the amount required to support its traffic. When the backhaul link is not congested, the LTE scheduler can prioritize users as usual, properly using radio and backhaul resources. However, when a "deficit" of bandwidth is detected, the scheduler should reduce the amount of traffic scheduled to nGBR bearers. In this way, the ONU-eNB buffer size can be controlled to prevent waste of both air interface and backhaul resources. If large amount of nGBR traffic arrives at the ONU-eNB buffer during congestion in the backhaul link, the delay of packets in the buffer will increase (undesirable effect in the network). Moreover, the buffer can overflow, thus wasting radio resources used to carry the dropped packets. The proposed method and the other components of the framework are described in [Astudillo 2015, Section 4.3].

Applying the method described above, modifications to the ZBQoS scheduler were introduced so that it can be aware of backhaul capacity. As a results, the Hybrid Z-Based QoS Scheduler (HZBQoS) was proposed. This scheduler represents the first mechanism in a new class of schedulers for LTE networks that we named backhaul-aware schedulers. The modifications introduced are described in [Astudillo 2015, Section 4.4]. 


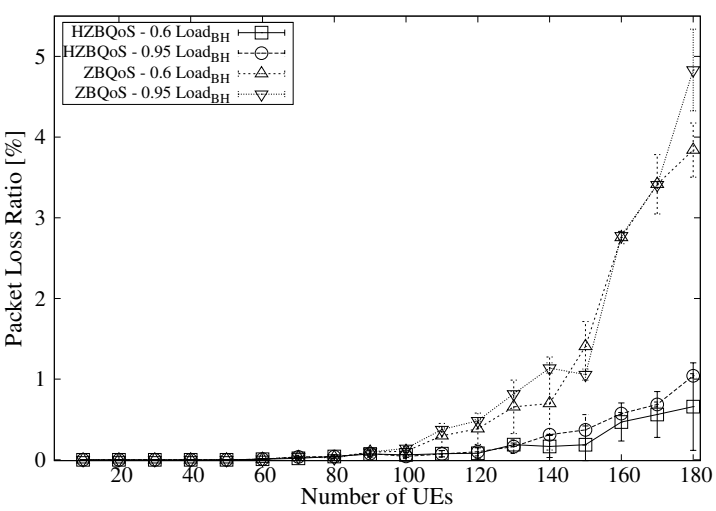

(a) Packet loss ratio for video traffic.

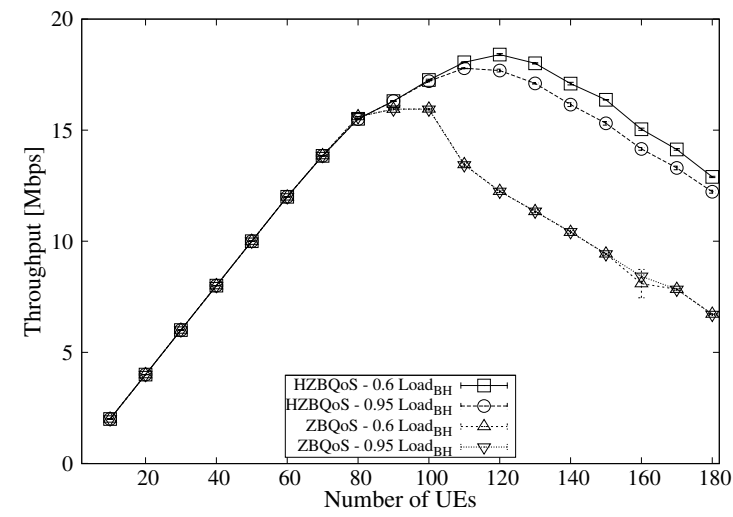

(b) Aggregated throughput for CBR traffic.

Figure 2. Performance of the HZBQoS scheduler

A simulator for LTE-EPON integrated networks was developed to assess the ability of the proposed framework to support QoS requirements of the LTE users and to improve performance under variable traffic conditions in both LTE and EPON network. We compared the performance of the scheduler modified with the proposed framework (HZBQoS) to that of the unmodified scheduler (ZBQoS).

The simulated scenarios use an EPON network with tree topology and optical channel capacity of $1 \mathrm{~Gb} / \mathrm{s}$. This network is composed of 1 Optical Line Terminal (OLT), 1 ONU-eNB and 31 conventional ONUs. The OLT employs the Interleaved Polling with Adaptive Cycle Time (IPACT) protocol with the limited scheduling policy [Kramer et al. 2002] for inter-ONU scheduling. Two distinct loads in the backhaul were used: 0.6 and 0.95 of the maximum EPON capacity, which correspond to a lightly loaded and a heavily loaded backhaul scenario, respectively. The LTE network part is composed of a single cell (served by the ONU-eNB device) and several users (varying from 10 to 180, with increments of 10). Table 4.1 and 4.2 in [Astudillo 2015] contain the settings in these simulations. The assumptions made in the evaluation of the ZBQoS scheduler hold here.

The HZBQoS scheduler is able to provide low packet loss ratio to video traffic under all traffic conditions in both the backhaul and the LTE network (Fig. 2a), which does not happen with the service provided by the ZBQoS scheduler. The PLR produced by ZBQoS surpasses $1 \%$ and increases with the traffic load, reaching $5 \%$ under heavy load.

The aggregated throughput of CBR users (Fig. 2b) shows the decrease of CBR traffic to meet the QoS requirements of real-time traffic in overloaded scenarios. However, peak throughput under the ZBQoS scheduler is achieved with 90 UEs while under the HZBQoS scheduler it is achieved with 120 UEs. Moreover, the throughput achieved by ZBQoS is independent of the backhaul load condition. This fact evinces the lack of capacity of the ZBQoS scheduler to deal with capacity variation of the backhaul link. The HZBQoS scheduler promotes a better use of network resources in scenarios with high load in both the backhaul and the mobile network, providing throughput $40 \%$ higher than that given by the ZBQoS scheduler. When Figures $2 \mathrm{a}$ and $2 \mathrm{~b}$ are analyzed together, the benefits the proposed framework brings are clear since the HZBQoS scheduler yields higher aggregated throughput while generating PLR values below $1 \%$ under highly loaded scenarios. More results are presented in [Astudillo 2015, Section 4.5]. 


\section{Publications}

Results in the dissertation [Astudillo 2015] were reported in four papers. Results on LTE uplink scheduling were published in the IEEE Globecom 2013 (Qualis A1) and the WRA 2014. Results on QoS provisioning in LTE-EPON networks were published in the IEEE WCNC 2014 (Qualis A2) and in the IEEE Wireless Communications (Qualis A1). In the 2014 Journal Citation Reports, this latter publication obtained the highest Impact Factor (6.524) in Computer Science, Telecommunications, and Electrical \& Electronic Engineering categories [Chen 2014]. In addition, we are preparing an article extending the results of the LTE scheduler that will be submitted to an international journal. Full references of the published papers can be found in Section 1.2 of the dissertation.

\section{Conclusion}

This article summarized the contribution of the master's thesis in [Astudillo 2015], which studied the QoS provisioning problem in LTE-EPON integrated networks. We introduced two novel uplink schedulers and a framework for QoS provisioning in these networks. The dynamic prioritization of requests and the consideration of backhaul information in radio resource allocation are original contributions of this work and they showed to increase the utilization of the network resources, while guaranteeing QoS requirements.

\section{References}

[Anas et al. 2008] Anas, M., Rosa, C., Calabrese, F., Pedersen, K., and Mogensen, P. (2008). Combined admission control and scheduling for QoS differentiation in LTE uplink. In VTC 2008-Fall, pages 1-5.

[Astudillo 2015] Astudillo, C. A. (2015). Provisão de qualidade de serviço em redes integradas LTE-EPON. Master thesis, LRC, Institute of Computing, UNICAMP.

[Chen 2014] Chen, H. (2014). Impact factor and open call articles [message from the editorin-chief]. IEEE Wireless Commun., 21(4):2-4.

[Chung et al. 2008] Chung, J., Park, J., Choi, B., Park, Y., Yoo, J., and Kim, B.-W. (2008). Application of 2W-PON to the backhaul of a wireless network. J. Optical Networking, 7:905.

[Cisco 2014] Cisco (2014). The zettabyte era: Trends and analysis. White Paper.

[Delgado and Jaumard 2010] Delgado, O. and Jaumard, B. (2010). Scheduling and resource allocation for multiclass services in LTE uplink systems. In WiMob, pages 355-360.

[Ghimire and Rosenberg 2014] Ghimire, J. and Rosenberg, C. (2014). Impact of limited backhaul capacity on user scheduling in heterogeneous networks. In WCNC 2014, pages 2480-2485.

[Kramer et al. 2002] Kramer, G., Mukherjee, B., and Pesavento, G. (2002). IPACT a dynamic protocol for an Ethernet PON (EPON). IEEE Commun. Mag., 40(2):74-80.

[Lim et al. 2012] Lim, W., Milosavljevic, M., Kourtessis, P., and John, M. (2012). QoS mapping for LTE backhauling over OFDMA-PONs. In ICTON, pages 1-4.

[Marwat et al. 2014] Marwat, S., Weerawardane, T., Zaki, Y., Goerg, C., and Timm-Giel, A. (2014). Analysis of radio resource allocation in LTE uplink. Wireless Personal Communications, 79(3):2305-2322. 